\title{
Physicochemical Properties of Polysaccharide Kefiran Isolated from Kefir Grains Biomass
}

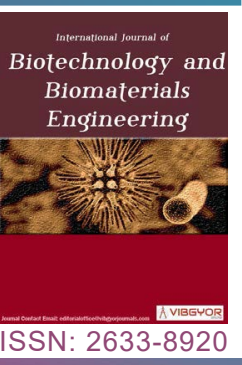

\section{Eda Ondul Koc ${ }^{1^{*}}$ and Mahmut Inal ${ }^{2}$}

${ }^{1}$ Food Processing Department, Food Quality Control and Analysis, Yeniçağa Yaşar Çelik Vocational School, Bolu Abant Izzet Baysal University, Bolu, Turkey

${ }^{2}$ Vegetable and Animal Production Department, Yüksekova Vocational School, Hakkari University, HAKKARI, Turkey

\begin{abstract}
Kefiran is a hydro-soluble polysaccharide, which can be isolated from kefir grains biomass. In the present study was characterised physicochemical and morphological properties of kefiran isolated from kefir grains biomass, obtaining from a local dairy company. High performance liquid chromatography analysis of monosaccharides revealed that kefiran was composed of glucose and galactose in an approximate ratio of 1.0:0.4 in nature. Infrared spectra of kefiran revealed the presence of carboxyl, hydroxyl, and amide groups, which corresponded to a typical heteropolymeric polysaccharide, which indicated a purified structure of isolated kefiran from kefir grains biomass. The scanning electron microscopy images of polysaccharide kefiran showed homogeneous morphology with porous and a spongelike structure. The functional groups of kefiran can make it more attractive for many modifications.
\end{abstract}

\section{Keywords}

Kefir grain, Kefiran, Infrared spectra, High performance liquid chromatography

\section{Introduction}

Kefiran, a hydro-soluble prebiotic branched exopolysaccharide, is a hydroxyl groups rich, and heteropolysaccharide biopolymer containing glucose and galactose [1-4]. Kefiran has a molecular weight of around $10^{6} \mathrm{Da}$ [5]. According to chemical structure of kefiran, it is a branched hexa- or hepta-saccharide repeating units, that itself is composed of a regular pentasaccharide unit to which one or two sugar residues are randomly attached. It had a backbone composed of $(1 \rightarrow 6)$-linked Glc, $(1 \rightarrow$ 3)-linked Gal, ( $1 \rightarrow 4)$-linked Gal, $(1 \rightarrow 4)$-linked Glc and $(1 \rightarrow 2,6)$-linked Gal, with branch attached to 0-2 of Gal residues and Glc residues situated in the structure end [2]. The diversity of kefiran glycosidic linkages is resistant to enzymatic degradation [3]. Kefiran is produced either from kefir grains or pure cultures kefiran of Lactobacillus kefiranofaciens under aerobic conditions or mixed cultures of Lactobacillus kefiranofaciens with Saccharomyces cerevisiae under anaerobic conditions [6-9].

Characteristics such as antimicrobial, anti-inflammatory, antitumor activities [10], immune-modulating, wound healing properties, blood pressure and cholesterol lowering ability, antioxidative,

\footnotetext{
*Corresponding author: Eda Ondul Koc, Food Processing Department, Food Quality Control and Analysis, Yeniçağa Yaşar Çelik Vocational School, Bolu Abant Izzet Baysal University, 14650 Yeniçağa/BOLU, Turkey Accepted: March 01, 2021; Published: March 03, 2021

Copyright: (C) $2021 \mathrm{Koc}$ EO, et al. This is an open-access article distributed under the terms of the Creative Commons Attribution License, which permits unrestricted use, distribution, and reproduction in any medium, provided the original author and source are credited.

Koc et al. Int J Biotechnol Biomater Eng 2021, 3:005
}

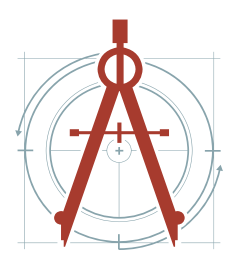


and anti-proliferative activities [11,12] have led to attract the attention of scientists. In addition to these, other functional properties of kefiran such as the ability to form edible films for use in food packaging $[13,14]$, a potential texture modifier as a hydrocolloid and Generally Recognized As Safe (GRAS) status [15-17] to improve rheological properties of acid milk gels due to its physicochemical properties in increasing viscosity and viscoelasticity [18] have been of interest to researchers.

Novel technologies have been used in recent years to extract bioactive compounds such as ultrasonic, microwave, supercritical fluid, and enzyme-assisted extraction [7]. Hot water extraction is the most commonly used method for extraction of microbial exopolysaccharides. In a variety of studies, hot water extraction has been used to extract kefiran from kefir grains [17-20]. The purpose of this study was to evaluate chemical composition, molecular structure and microscopic morphology of the extracted kefiran and to examine the characteristics by applying different methodologies such as Fourier transform infrared spectroscopy, high performance liquid chromatography and scanning electron microscopy. These characteristics could help us to identify potential application for food industry and present some important basis for kefiran that has several health promoting properties.

\section{Materials and Methods}

\section{Fermentation conditions}

Kefir grains were purchased from a local dairy company, Turkey. The grains were reactivated in ultra high temperature treated skimmed milk obtained from a market. Kefir grains (10 g) were inoculated into $100 \mathrm{~mL}$ of milk. After incubation at $20{ }^{\circ} \mathrm{C}$ for $24 \mathrm{~h}$, the grains were seperated by filtration from fermented product. The medium was exchanged at the end of incubation for new culture. The culture fermentation was repeated for four subsequent weeks to increase kefir grains biomass.

\section{Extraction of kefiran biopolymer}

The isolation and purification of kefiran biopolymer was achieved employing a combination of processing steps as described by Piermaria, et al. [19]. The method involved heating of kefir grains in a erlen mayer boiling water at $100^{\circ} \mathrm{C}$ placed on a magnetic stirrer hot plate at a ratio 1:10 (kefir grain:water) and stirred $30 \mathrm{~min}$ (Arec Heating Magnetic
Stirrer). The mixture was centrifuged at 10.000 $\mathrm{g}$ for $20 \mathrm{~min}$ at $20^{\circ} \mathrm{C}$ (Universal $32 \mathrm{R}$ by Hettich, Tuttlingen-Germany). The supernatant was precipited by addition of two volumes of cold ethanol and kept at $-20{ }^{\circ} \mathrm{C}$ overnight. Precipitation phases were repeated two times. Finally, the kefiran exopolysaccharide was left as a white precipitate, and freeze dried (Labconco, USA). Chemical hydrolysis of kefiran polysaccharide was performed based on the method of Skalska-Kamińska, et al. [21]. The complete acid hydrolysis of kefiran was carried out using a heating plate. The determination of sugar residues obtained by chemical hydrolysis was performed by thin layer chromatography (TLC) and high performance liquid chromatography (HPLC) analyses.

\section{Monosaccharides identification by TLC}

The composition of acid hydrolyzed kefiran polysaccharide was investigated using the method of Adigüzel and Tunçer, [22]. The mobile phase was used as a mixture of formic acid, butanone, tert butanole, water (15:30:40:15 v/v/v/v), the spots were visualized by spraying aniline ( $2 \% \mathrm{v} / \mathrm{v})$, phytallic acid $(3.5 \% \mathrm{w} / \mathrm{v})$ in water-saturated butanol and baking in oven at $90{ }^{\circ} \mathrm{C}$ for $5 \mathrm{~min}$. The stock solutions for standards were prepared by dissolving $0.01 \mathrm{~g}$ of D-glucose and D-galactose (purchased from Sigma-Aldrich) in $100 \mathrm{~mL}$ distilled water.

\section{Measurement of total sugars composition}

Phenol-sulfuric acid method was used to estimate total sugars of the extracted kefiran samples with glucose as standard. $2 \mathrm{mg}$ kefiran sample (1.0 $\mathrm{mg} / \mathrm{mL}$ ) was mixed with $1 \mathrm{~mL}$ phenol solution (5\%) and $5 \mathrm{~mL}$ concentrated sulfuric acid at $25^{\circ} \mathrm{C}$ for 5 $\min$. The reaction solution was then heated for 15 min in boiling water bath. Absorbance was read at $485 \mathrm{~nm}$ by UV-visible spectrophotometer (UV mini1240, Shimadzu-Europe) [7].

\section{Monosaccharide composition of the kefiran}

High performance liquid chromatography analysis was performed to check monosaccharide composition of the kefiran sample [23]. The kefiran solutions in a ratio $1: 10(\mathrm{w} / \mathrm{w})$, which were extracted and hydrolysed according to the method described above, then all the samples were filtered through a microfilter of pore size of 0.45 $\mathrm{mm}$ (Millipore, Billerica, Massachusetts, USA) to remove impurities. The monosaccharide profile was 
determined by HPLC on a Agilent system (Agilent, Barcelona, Spain) equipped by measuring the refractive index detector (RID 20A device). The HPLC column was $\mu$ bondapak- $\mathrm{NH}_{2}$ column, $250 \mathrm{~mm} \times 4.6$ $\mathrm{mm}$, particle size $5 \mu \mathrm{m}$. The column was set at $30^{\circ} \mathrm{C}$ using a temperature controller (Agilent) for separations. The chromatographic separation was carried at a flow rate of $1.3 \mathrm{~mL}$. $\mathrm{min}^{-1}$, using a mixture aceto nitrile:water 80:20 (v/v) as a mobile phase. Standard glucose and galactose (Sigma, $\geq 99 \%$ ) were prepared for comparison [24].

\section{Measurement of protein content}

Bradford method was used to measure protein content of the kefiran samples with bovine serum albumin as standard. $1.0 \mathrm{~mL}$ kefiran sample $(1 \mathrm{mg} /$ $\mathrm{mL}$ ) was incubated with $5 \mathrm{~mL}$ Coomassie Brilliant Blue $\mathrm{G} 250$ at $25^{\circ} \mathrm{C}$ for $15 \mathrm{~min}$. Absorbance was read at $595 \mathrm{~nm}$ by UV-visible spectrophotometer [25].

\section{Fourier transform infrared spectroscopy}

Chemical structure of kefiran polysaccharide was investigated by Fourier transform-infrared spectroscopy (FT-IR). The infrared spectroscopy range of the samples was obtained in the wavenumber of $400-4000 \mathrm{~cm}^{-1}$ through FTIR (Model Vertex 70v, Brucker, Germany) [26].

\section{Scanning electron microscope}

The kefiran solutions were cryofixed by immersion in liquid nitrogen and immediately freeze dried. The morphology images of all samples were investigated by scanning electron microscopy (Model Carl Zeiss EVO LS 10 SEM, Germany) at an accelerating voltage of $20 \mathrm{kV}$. The samples were coated with a layer of gold, $20 \mathrm{~nm}$ thick before SEM examination [19].

\section{Results and Discussion}

\section{Evaluation of kefiran polysaccharide composi- tion}

According to method reported by Adıgüzel and Tunçer, [22] sugar composition of kefiran EPS carried out by TLC that showed the presence of glucose and galactose and was in agreement with previous results $[19,27]$. As depicted in Figure 1 , in acid hydrolyzed kefiran and standard sugars was identified as good shape spots on TLC plate. Non-hydrolyzed kefiran seperation was not achieve because the kefiran has high molecular weight $[19,28]$. Glucose and galactose monosaccharides in the hydrolized kefiran were visualized on the same spot on TLC plate due to similar molecular weights. The total sugar content of kefiran was estimated as $93.0 \%$ by the phenol-sulfuric acid method. In similar studies, total sugar contents of kefiran extracted by hot water were 99\% [29] and $86.55 \%$ [7]. As determined by Bradford protein assay, kefiran grain showed a high purity grade (data not shown). Piermaria, et al. ([19] reported concentration of less than $0.01 \%$ of protein expressed per dry matter in kefiran solution. Indeed, kefir grains compose of a polysaccharide known as kefiran and a protein matrix [30]. The sugar composition of kefiran exopolysaccharide was analyzed using high performance liquid chromatography (Figure 2). The results from this study are given which revealed that kefiran is composed of glucose and galactose in a relative molar ratio of 1.0:0.4. Glucose had the higher proportion than galactose. This is different from that of the exopolysaccharide produced by kefir grain that consists of approximately equal proportions of galactose and glucose residues. Also, other researchers $[13,27,31-39]$ have shown that the kefiran polysaccharide contains approximately equal amounts of glucose and galactose. In this work, these data are in agreement with the results reported by Liu, et al. [5]. He reported that the polysaccharide ke-

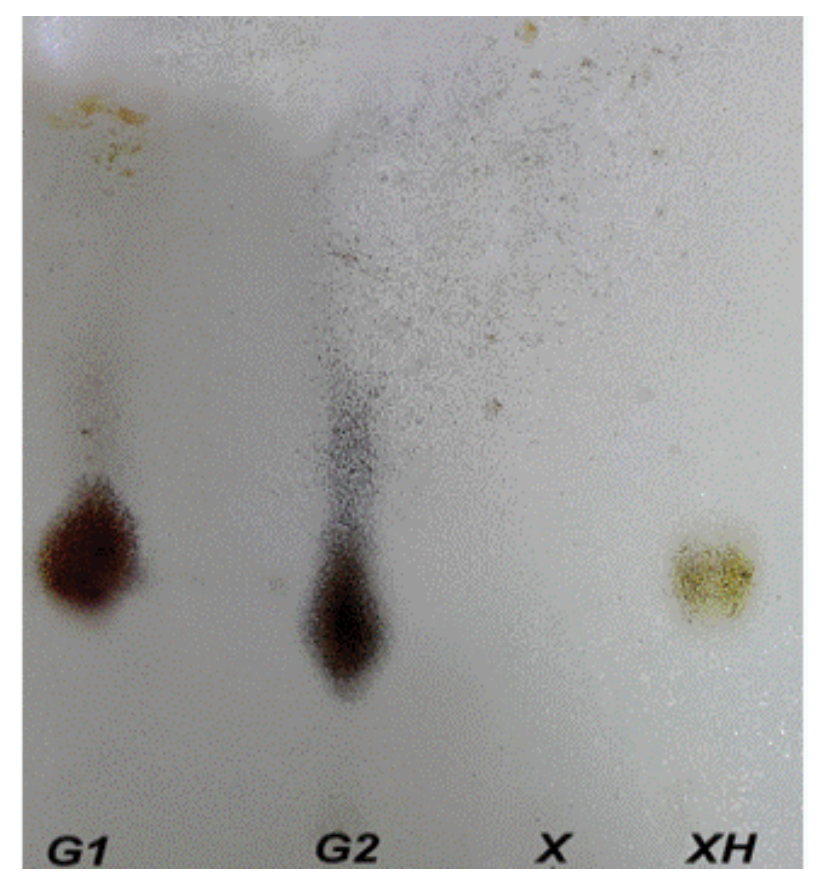

Figure 1: TLC chromatogram of the hydrolyzed kefiran sample and standards. G1, G2: D-Glucose, D-Galactose standards, X: Non-hydrolyzed kefiran; $\mathrm{XH}$ : Hydrolyzed kefiran. 


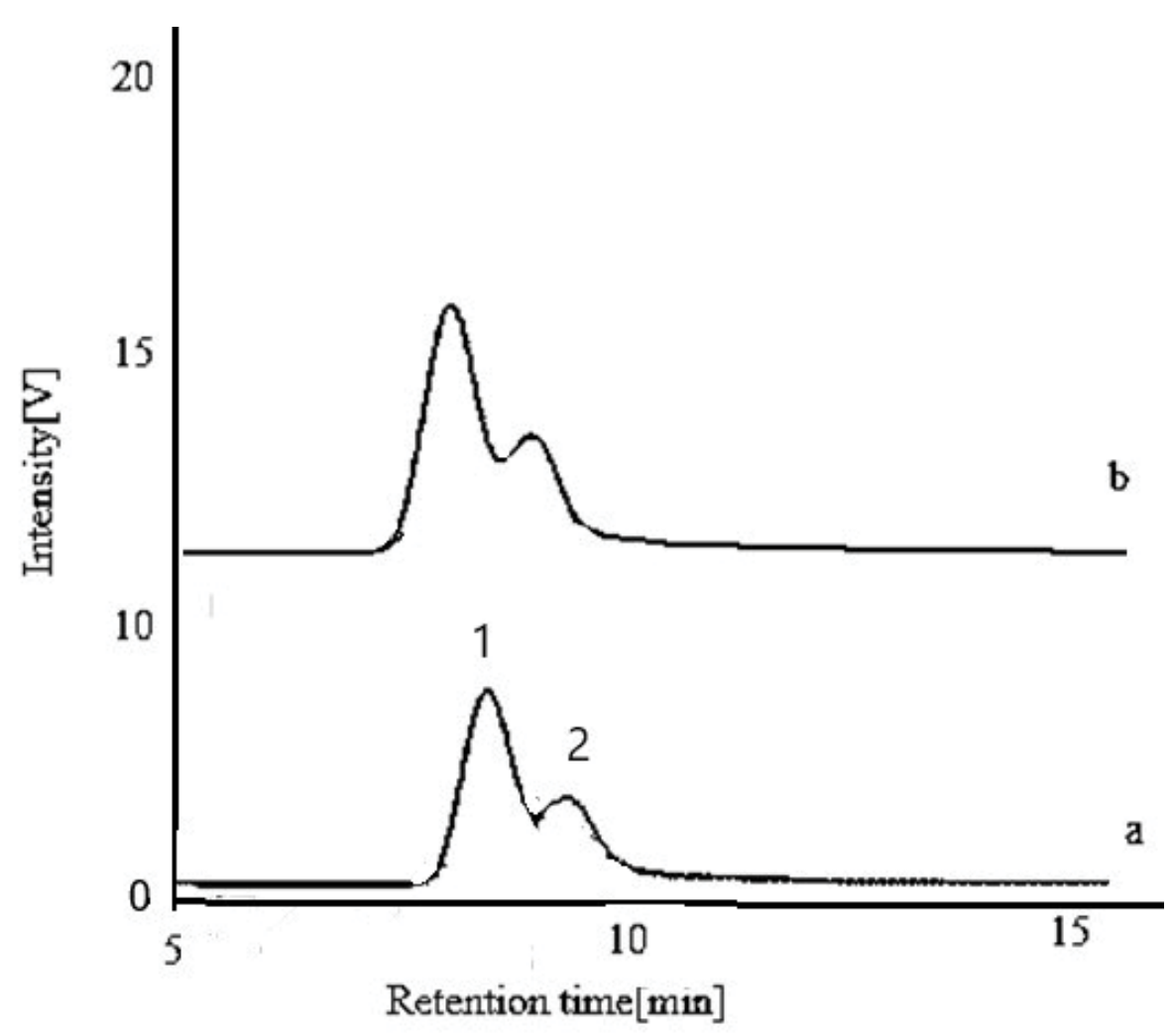

Figure 2: HPLC chromatogram of monosaccharides identified in $0.2 \mathrm{~g} \cdot \mathrm{ml}^{-1}$ kefiran solution. Standard mix of glucose and galactose (a) Kefiran solution;1-glucose with retention time 7.262, 2-galactose with retention time 7.877 (b).

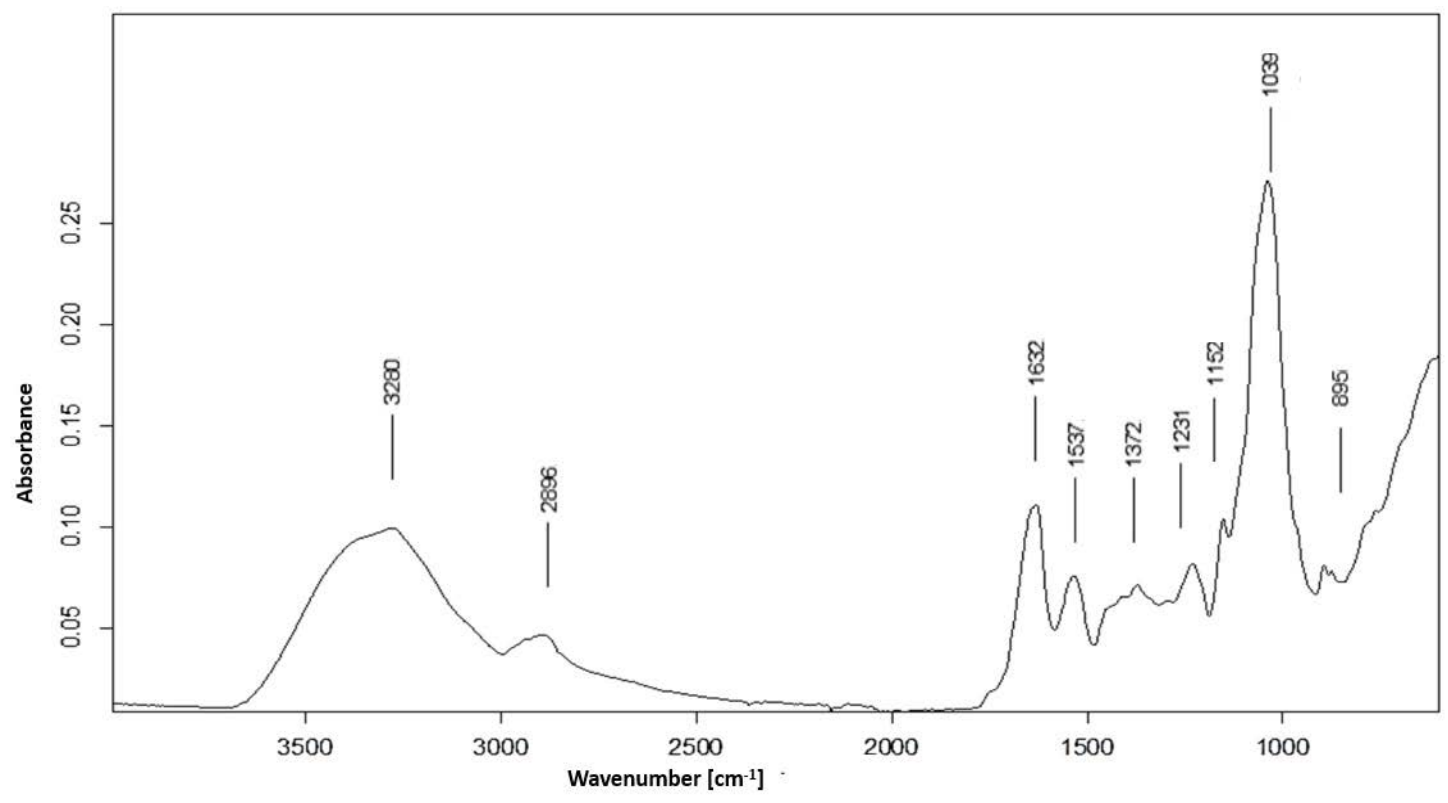

Figure 3: FT-IR spectra of prebiotic kefiran exopolysaccharide.

firan isolated from kefir grains grown in soymilk composed of more glucose than galactose. It has been explained that growth conditions and medium composition affect the sugar composition of the exopolysaccharide produced by lactic acid bac- teria. Zajsek, et al. [40] identified glucose and galactose residues within the kefiran samples in a ratio of 1:0.7 using capillary electrophoresis. Pop, et al. [29] reported that kefiran polysaccharide isolated from kefir grains biomass is composed of glucose 
and galactose in a relative molar ratio of 0.94:1.1. Wang, et al. [41] reported that EPS produced by Lactobacillus plantarum KF5 was composed of mannose, glucose and galactose in a approximate ratio 1:4.99:6.90. Exopolysaccharides of lactic acid bacteria is used mainly in fermented milk technology may consist glucose, fructose, galactose, rhamnose, xylose, mannose [42].

\section{Fourier transform infrared spectroscopy (FT-IR)}

The FT-IR results of kefiran exopolysaccharide demonstrated peaks with various characteristics within the wavenumber range from 3400 to 850 $\mathrm{cm}^{-1}$ (Figure 3). A major absorbtion peak $3280 \mathrm{~cm}^{-1}$ was observed, which was indicative of hydroxyl groups attributed to polysaccharides and water $[29,41,43]$. A weak stretching peak at $2896 \mathrm{~cm}^{-1}$ showed the presence of methyl groups in kefiran. The regio $1775-1700 \mathrm{~cm}^{-1}$ assigned to peaks contain lipid components in kefiran. Strong absorption at $1632 \mathrm{~cm}^{-1}$ was also showed which corresponded to amide $\mathrm{I}>\mathrm{C}=\mathrm{O}$ stretch and $\mathrm{C}-\mathrm{N}$ bending of protein and peptide amines [7]. The finger print region for carbohydrates included the range from 1200 to $900 \mathrm{~cm}^{-1}$ which associated with stretching modes of carbohydrate rings and side group (-C-O-C, $\mathrm{C}-\mathrm{OH}$ and $\mathrm{C}-\mathrm{H}$ ). The peaks around $900 \mathrm{~cm}^{-1}$ were indicative of the vibration modes of glucose, galactose and $\beta$-linkage in the kefiran structure $[2,28,31,44]$.

\section{Scanning electron microscope (SEM)}

In our research, the surface morphology of the kefiran were evaluated by SEM. SEM analysis of kefiran cryogels showed homogeneous morphology with porous and a sponge-like structure (Figure $4 \mathrm{a}$ and Figure $4 \mathrm{~b})$. This images were evaluated as a highly porous material, the unique microstructure of the kefiran exopolysaccharide makes it remarkable among common materials due to its distingue characteristics. Piermaria, et al. [19] reported that when the kefiran solution is allowed to gel before freeze drying quite a different structure is obtained. This a three-dimensional sponge like structure explains the high water holding capasity of the gels.

\section{Conclusion}

This paper presented isolation and physicochemical characterization of kefiran exopolysaccharide produced by kefir grains obtaining from a local dairy company. The results of structure identification indicated that kefiran polysaccharide contains glucose and galactose in a ratio of 1.0:0.4. Infrared spectra indicated very important reactive functional groups of kefiran, which can make it more flexible for many modifications. Sugar compositions of hydrolyzed kefiran exopolysaccharide achieved by TLC showed the presence of glucose and galactose. The total sugar content of kefiran was estimated as $93.0 \%$ by the phenol-sulfuric acid method.

\section{Acknowledgements}

The authors gratefully acknowledge the financial support provided by Yüzüncü Yıl University Sci-
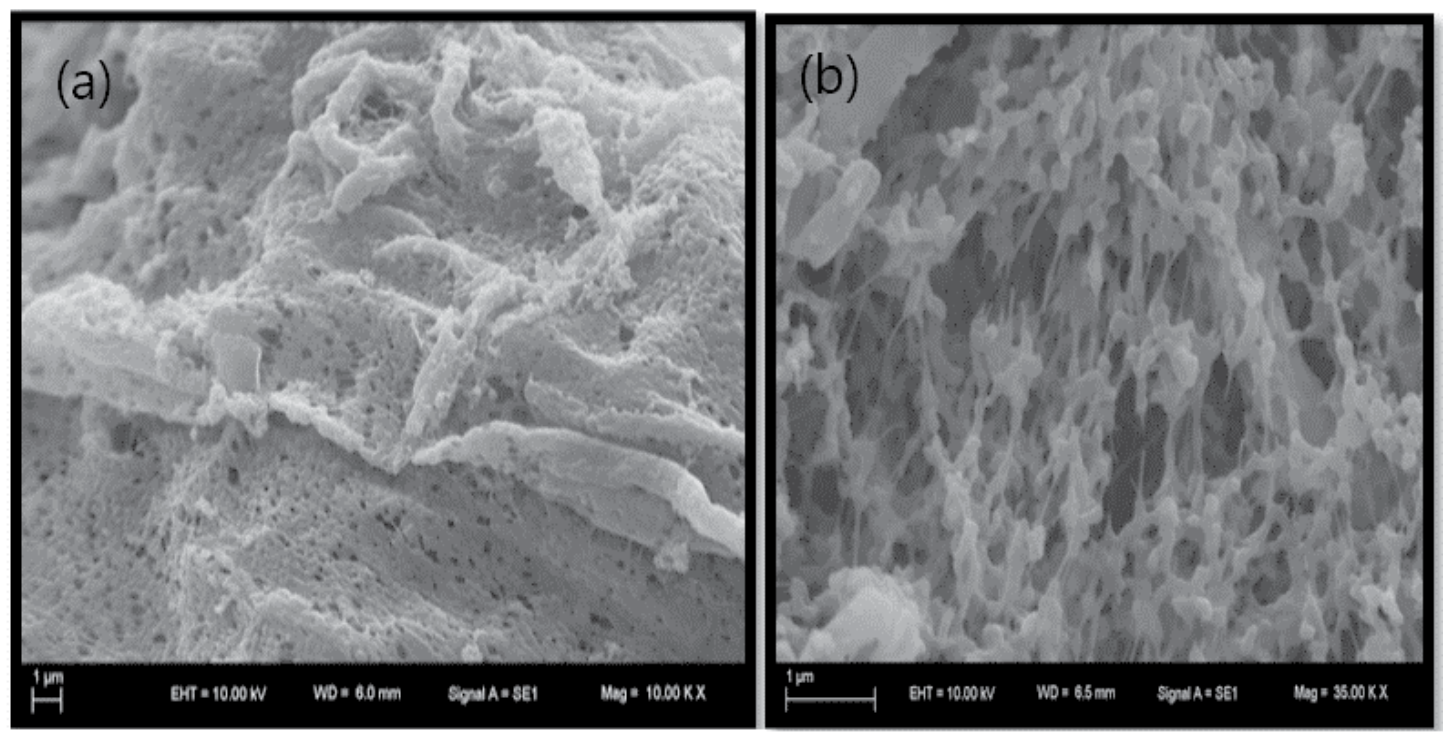

Figure 4: The microstructure and surface morphology micrographs of kefiran cryogels (placed in a freezer at $-20^{\circ} \mathrm{C}$ for $24 \mathrm{~h}$ and transferred to a refrigerator at $4{ }^{\circ} \mathrm{C}$ for a further $24 \mathrm{~h}$ ) [17] observed by SEM a) 10.00 $\mathrm{Kx}$; b) $35.00 \mathrm{Kx}$. 
entific Research Projects Department, Van, Turkey (Project No:2015-FBE-YL).

\section{References}

1. Moradi Z, Esmaiili M, Almasi H (2019) Development and characterization of kefiran - $\mathrm{Al}_{2} \mathrm{O}_{3}$ nanocomposite films: Morphological, physical and mechanical properties. Int J Biol Macromol 122: 603-609.

2. Moradi Z, Kalanpour N (2019) Kefiran, a branched polysaccharide: Preparation, properties and applications: A review. Carbohydrate Polymers 223: 115100.

3. Xu Y, Cui Y, Yue F, Liu L, Shan Y, et al. (2019) Exopolysaccharides produced by lactic acid bacteria and Bifidobacteria: Structures, physiochemical functions and applications in the food industry. Food Hydrocolloids 94: 475-499.

4. Hsieh HH, Wang SY, Chen TL, Huang YL, Chen MJ (2012) Effects of cow's and goat's milk as fermentation media on the microbial ecology of sugary kefir grains. International Journal of Food Microbiology 157: 73-81.

5. Liu JR, Chen MJ, Lin CW (2002) Characterization of polysaccharide and volatile compounds produced by kefir grains grown in soymilk. J Food Sci 67: 104-108.

6. Badel S, Bernardi T, Michaud P (2011) New perspectives for Lactobacilli exopolysaccharides. Biotechnology Advances 29: 54-66.

7. Hasheminya SM, Dehghannya J (2020) Novel ultrasound-assisted extraction of kefiran biomaterial, a prebiotic exopolysaccharide, and investigation of its physicochemical, antioxidant and antimicrobial properties. Materials Chemistry and Physics 243: 122645.

8. Cheirsilp B, Shimizu H, Shioya S (2007) Kinetic modeling of kefiran production in mixed culture of Lactobacillus kefiranofaciens and Saccharomyces cerevisiae. Process Biochemistry 42: 570-579.

9. Tada S, Katakura Y, Ninomiya K, Shioya S (2007) Fed-batch coculture of Lactobacillus kefiranofaciens with Saccharomyces cerevisiae for effective production of kefiran. Journal of Bioscience and Bioengineering 103: 557-562.

10.Barbosa AF, Santos PG, Lucho AMS, Schneedorf JM (2011) Kefiran can disrupt the cell membrane through induced pore formation. Journal of Electroanalytical Chemistry 653: 61-66.

11.Saadat YR, Khosroushahi AY, Gargari BP (2019) A comprehensive review of anticancer, immunomodulatory and health beneficial effects of the lactic acid bacteria exopolysaccharides. Carbohydrate Polymers 217: 79-89.
12.Chen Z, Shi J, Yang X, Nan B, Liu Y, et al. (2015) Chemical and physical characteristics and antioxidant activities of the exopolysaccharide produced by Tibetan kefir grains during milk fermentation. International Dairy Journal 43: 15-21.

13.Gagliarini N, Diosma G, Garrote GL, Abraham AG, Piermaria J (2019) Whey protein-kefiran films as driver of probiotics to the gut. LWT 105: 321-328.

14. Ghasemlou M, Khodaiyan F, Oromiehie A, Yarmand MS (2011) Characterization of edible emulsified films with low affinity to water based on kefiran and oleic acid. International Journal of Biological Macromolecules 49: 378-384.

15.Piermaría J, Bengoechea C, Abraham AG, Guerrero A (2016) Shear and extensional properties of kefiran. Carbohydr Polym 152: 97-104.

16. Radhouani H, Bicho D, Gonçalves C, Maia FR, Reis RL, et al. (2019) Kefiran cryogels as potential scaffolds for drug delivery and tissue engineering applications. Materials Today Communications 20: 100554.

17. Exarhopoulos S, Raphaelides SN, Kontominas MG (2018) Flow behavior studies of kefiran systems. Food Hydrocolloids 79: 282-290.

18.Piermaria JA, Pinotti A, Garcia MA, Abraham AG (2009) Films based on kefiran, an exopolysaccharide obtained from kefir grain: Development and characterization. Food Hydrocolloids 23: 684-690.

19.Piermaria JA, de la Canal ML, Abraham AG (2008) Gelling properties of kefiran, a food-grade polysaccharide obtained from kefir grain. Food Hydrocolloids 22: 1520-1527.

20. Ghasemlou M, Khodaiyan F, Oromiehie A (2011) Rheological and structural characterisation of film-forming solutions and biodegradable edible film made from kefiran as affected by various plasticizer types. International Journal of Biological Macromolecules 49: 814-821.

21.Skalska-Kamińska A, Matysik G, Wójciak-Kosior M, Donica H, Sowa I (2009) Thin-layer chromatography of sugars in plant material. Annales Universitatis Mariae Curie-Sklodowska, Sectio DDD: Pharmacia 22: 17-24.

22.Adigüzel AO, Tunçer $M$ (2015) Hydrolysis of pretreated lignocellulosic wastes by streptomyces $\mathrm{Sp}$. AOA40 isolated from mersin for total reducing sugars. International Journal of Advanced Biotechnology and Research(IJBR) 6: 372-382.

23. Shukla A, Mehta K, Parmar J, Pandya J, Saraf M (2019) Depicting the exemplary knowledge of microbial exopolysaccharides in a nutshell. European Polymer Journal 119: 298-310. 
24. Melgarejo P, Salazar DM, Artés F (2000) Organic acids and sugars composition of harvested pomegranate fruits. European Food Research and Technology 211: $185-190$.

25.Bradford MM (1976) A rapid and sensitive method for the quantitation microgram quantities of protein utilizing the principle of protein-dye binding. Anal Biochem 72: 248-254.

26.Shang N, Xu R, Li P (2013) Structure characterization of an exopolysaccharide produced by Bifidobacterium animalis RH. Carbohydr Polym 91: 128-134.

27. Pop C, Rotar AM, Salanță LC, Semeniuc CA, Socaciu C, et al. (2015) Fingerprint profiling of polysaccharide kefiran extracted from kefir grains biomass. Journal of Agroalimentary Processes and Technologies 21: 207-212.

28.Ghasemlou M, Khodaiyan F, Jahanbin K, Gharibzahedi SMT, Taheri S (2012) Structural investigation and response surface optimisation for improvement of kefiran production yield from a low-cost culture medium. Food Chemistry 133: 383-389.

29.Pop CR, Salanţă L, Rotar AM, Semeniuc CA, Socaciu C, et al. (2016) Influence of extraction conditions on characteristics of microbial polysaccharide kefiran isolated from kefir grains biomass. Journal of Food and Nutrition Research 55: 121-130.

30. Leite AMO, Leite DCA, Del Aguila, EM, Alvares TS, Peixoto RS, et al. (2013) Microbiological and chemical characteristics of Brazilian kefir during fermentation and storage processes. Journal of Dairy Science 96: 4149-4159.

31.Santos A, San Mauro M, Sanchez A, Torres JM, Marquina D (2003) The antimicrobial properties of different strains of Lactobacillus spp. isolated from kefir. Systematic and Applied Microbiology 26: 434-437.

32.Ghasemlou M, Khodaiyan F, Oromiehie A, Yarmand MS (2011) Development and characterisation of a new biodegradable edible film made from kefiran, an exopolysaccharide obtained from kefir grains. Food Chemistry 127: 1496-1502.

33. Sabaghi M, Maghsoudlou Y, Habibi P (2015) Enhancing structural properties and antioxidant activity of kefiran films by chitosan addition. Food Structure 5: 66-71.

34.Blandón LM, Noseda MD, Islan GA, Castro GR, de Melo Pereira GV, et al. (2018) Optimization of cul- ture conditions for kefiran production in whey: The structural and biocidal properties of the resulting polysaccharide. Bioactive Carbohydrates and Dietary Fibre 16: 14-21.

35.Dadashi S, Boddohi S, Soleimani N (2019) Preparation, characterization, and antibacterial effect of doxycycline loaded kefiran nanofibers. Journal of Drug Delivery Science and Technology 52: 979-985.

36.Zajšek K, Goršek A, Kolar M (2013) Cultivating conditions effects on kefiran production by the mixed culture of lactic acid bacteria imbedded within kefir grains. Food Chem 139: 970-977.

37. Rimada S, Abraham AG (2006) Kefiran improves rheological properties of glucono- $\delta$-lactone induced skim milk gels. Int Dairy J 16: 33-39.

38. Toscano M, Carfi PF, Conoscenti G, Sabatino MA, La Carrubba V, et al. (2018) Kefiran-based scaffolds for biomedical applications. Chem Eng Trans 64: 181-186.

39. Wang Y, Ahmed Z, Feng W, Li C, Song S (2008) Physicochemical properties of exopolysaccharide produced by Lactobacillus kefiranofaciens ZW3 isolated from Tibet kefir. Int J Biol Macromol 43: 283-288.

40.Zajšek K, Kolar M, Goršek A (2011) Characterisation of the exopolysaccharide kefiran produced by lactic acid bacteria entrapped within natural kefir grains. Int J Dairy Technol 64: 544-548.

41. Wang Y, Li C, Liu P, Ahmed Z, Xiao P, et al. (2010) Physical characterization of exopolysaccharide produced by Lactobacillus plantarum KF5 isolated from Tibet Kefir. Carbohydrate Polymers 82: 895-903.

42. Enikeev R (2012) Development of a new method for determination of exopolysaccharide quantity in fermented milk products and its application in technology of kefir production. Food Chemistry 134: 2437-2441.

43. Hosseini SS, Khodaiyan F, Seyed SM, Azimi SZ, Gharaghani $M$ (2019) Immobilization of pectinase on the glass bead using polyaldehyde kefiran as a new safe cross-linker and its effect on the activity and kinetic parameters. Food Chemistry 309: 125777.

44.Zhao G, Kan J, Li Z, Chen Z (2005) Structural features and immunological activity of a polysaccharide from Dioscorea opposita Thunb roots. Carbohydrate Polymers 61: 125-131. 